\title{
Design of Bi-functional Bioconjugated Gold Nanoparticles by Pulsed Laser Ablation with Minimized Degradation
}

\author{
Annette BARCHANSKI ${ }^{* 1}$, Csaba Laszlo SAJTI ${ }^{1}$, Camilla SEHRING $^{1}$, Svea PETERSEN ${ }^{1}$ and Stephan BARCIKOWSKI ${ }^{2}$ \\ ${ }^{1}$ Laser Zentrum Hannover e.V., Nanotechnology Department, Hollerithallee 8, \\ 30419 Hannover, Germany \\ ${ }^{2}$ University of Duisburg-Essen, Institute of Technical Chemistry, Universitätsstraße 7, 45141 Essen, \\ Germany \\ E-mail: stephan.barcikowski@uni-due.de
}

\begin{abstract}
Femtosecond-pulsed laser ablation of gold is investigated in electron donor moiety containing biomolecule solutions, resulting in the controlled design of biocompatible, partially charged and electrostatic-stabilized gold nanoconjugates by in situ surface functionalization. The cell-penetrating peptide penetratin and a targeting miniStrep aptamer sequence are conjugated simultaneously to a single nanoparticle for the first time, producing highly stable gold nanoparticle bi-conjugates with defined degrees of surface coverages for each biomolecule. Ablating gold by $100 \mu \mathrm{J}$ laser pulses in presence of $1 \mu \mathrm{M}$ penetratin and $4.5 \mu \mathrm{M}$ miniStrep yields an average bioconjugate size of $10 \mathrm{~nm}$ and results in $61 \mathrm{pmol} \mathrm{cm}^{-2}$ surface coverage for the aptamer and $30 \mathrm{pmol} \mathrm{cm}^{-2}$ for the penetratin. Whereat, degree of surface coverage shows direct correlation to process parameters as well as to biomolecule size, concentration and steric dimension of the biomolecules used. To investigate the influence of time-delayed bioconjugation in view of more efficient up-scaling process, a fast ex situ conjugation is presented in a continuous flow chamber secondary, allowing highly controlled bioconjugation without irradiating the photo- and thermal-sensitive biomolecules and permitting the use of nanosecond laser pulses.

DOI:10.2961/jlmn.2011.02.0006
\end{abstract}

Keywords: cell-penetrating peptide, aptamer, ultrashort-pulsed laser ablation, surface coverage, in situ conjugation, fast ex situ conjugation

\section{Introduction}

Over the last years, the demand on specifically designed bio-nanoconjugates has increased significantly for biomedical applications. As it has become popular to use different features combined within a single conjugate, the controlled multi-functionalization of nanoparticles is essential.

Gold nanoparticles (AuNP) are attractive for biomedical use, due to their quantum dots surmounting biocompatibility [1]. Furthermore, biocompatible AuNP exhibit exceptional optical properties as a result of enhanced surface plasmon absorption and scattering [2, 3], enabling diverse imaging and mapping possibilities. On the other hand, owing to the ease of AuNP surface functionalization, applications like cell-targeted drugdelivery $[4,5]$ and epitope labeling $[6,7]$ are facilitated when coupled to diverse bioactive molecules as cellpenetrating peptides [8-10], oligonucleotides [11], aptamers [7, 12], siRNA [13] or drugs [14, 15].

Conventional AuNP generation method is based on standard chemical synthesis, covering the reduction of $\mathrm{Au}(\mathrm{III})[16,17]$. As cytotoxic chemical preservatives and reducing agents are involved during this long-termed reaction process, AuNP fabrication by ultrashort-pulsed laser ablation in liquids $[18,19]$ can qualitatively compete to this general particle synthesis route. Various metals ( $\mathrm{Au}, \mathrm{Ag}, \mathrm{Cu}$, etc.) ceramics (e.g., $\mathrm{ZrO}_{2}, \mathrm{Al}_{2} \mathrm{O}_{3}$ ) or alloys may be used, resulting in ligand-free nanoparticles within the stable handling form of a colloid [20].

The AuNP generated by this method are partially charged, increasing their electrostatic stability and offering the feature of in situ functionalization subsequently to fabrication process by molecules containing electron-donor moieties (e.g. thiol, disulfide) [11].

During in situ functionalization, the typical broad size distribution of ligand-free gold nanoparticles is quenched to a mean Feret diameter of 5 to $8 \mathrm{~nm}$, as determined in previous work [11]. The degree of biomolecule integrity was also analyzed in this work for different pulse energies and focal positions and it was found that $100 \mu \mathrm{J}$ laser pulses do not induce significant degradation to oligonucleotides.

Cell-penetrating peptides (CPPs) are useful tools for the intracellular delivery of cargo molecules like polypeptides, and nucleic acids [21] or even nanoparticles [22] to cells in culture in an energy-independent manner. Each CPP features a distinct protein transduction domain which promotes the translocation across biological membranes.

One of the most studied CPPs is penetratin, derived from the Drosophila Antennapedia homeodomain (amino acids 43-58: RQIKIWFQNRRMKWKK), which is internalized into cells in culture by receptor-independent uptake [23], endocytotic uptake [24] or the transient formation of inverted micelles [25] depending on the attached cargo molecule. Another prominent CPP termed TAT (transactivator of transcription; amino acids 47-57: GRKKRRQRRR) is expressed by the human immunodeficiency virus type-1 (HIV-1). TAT is involved in transcription and able to cross the plasma membrane of cells and to localize in the nucleus [26]. This mechanism was used recently by de la Fuente et al. for the nuclear delivery of TAT-conjugated gold nanoparticles [27]. 
Moreover, Petersen et al. achieved the successful conjugation of penetratin [10] and Sajti et al. presented TAT-conjugation [28] to laser-generated AuNP whilst both demonstrated the supporting effect of the CPP on the cellular penetration of AuNP.

In last years, aptamers got in the focus of interest of biotechnology as these short, single-stranded DNA or RNA molecules exhibit high affinity and specificity towards their complementary targets. Aptamer-conjugated AuNP have already been successfully applied for different biosensor detection strategies [29-31] and hence are interesting candidates for specific in-vitro targeting applications. For example, the application of an aptamer against streptavidin (referred to as miniStrep) in protein microarray was previously reported by Walter et al. [32]. Moreover, the in situ conjugation of miniStrep to laser-generated gold nanoparticles was demonstrated recently [12].

The individual combination of different bioactive features like cellular penetration and specific molecule targeting on a single nanoparticle can be achieved by controlled laser-induced in situ multi-functionalization with diverse biomolecules in a liquid. In the present work, we investigate the bi-functionalization of laser-generated gold nanoparticles with the CPP penetratin and the antistreptavidin aptamer miniStrep simultaneously, in terms of conjugation efficiency and controllable surface coverage.

According to literature, femtosecond laser irradiation minimize the thermal load both to matter and environment as the electron-phonon coupling occurs in a longer timescale that the irradiation. However, we recently investigated that even ultrashort laser pulses can significantly degrade sensitive biomolecules due to laser beam absorption of the generated colloidal nanohybrids, whereas use of continuous liquid flow drastically increased integrity [33]. Thus, besides in situ conjugation, a novel method to generate bio-functionalized gold nanoparticles with $100 \%$ integrity is investigated by fast ex situ conjugation in liquid flow. During this process, biomolecules are not irradiated and conjugation takes place subsequently to nanoparticle generation via laser ablation by using a custom-built flow chamber.

\section{Experimentals}

\subsection{Fabrication of AuNP}

AuNP were generated by pulsed laser ablation in double distilled aqueous solution $\left(\mathrm{ddH}_{2} \mathrm{O}, 18 \mathrm{M} \Omega\right)$. Laser ablation was performed on a gold target $(5 \mathrm{~mm} \times 5 \mathrm{~mm} \times 0.1 \mathrm{~mm}$, purity $>99.99 \%$ purchased from Goodfellow $\mathrm{GmbH}$ ) by femtosecond laser illumination (Spitfire Pro, Spectra Physics) delivering $120 \mathrm{fs}$ laser pulses at a wavelength of $800 \mathrm{~nm}$ (maximum pulse energy of $400 \mu \mathrm{J}$ with beam diameter of $4 \mathrm{~mm}$ ). A stable, red-colored colloidal solution was obtained using pulse energy of $100 \mu \mathrm{J}$ and a repetition rate of $5 \mathrm{kHz}$ with spot size of $250 \mu \mathrm{m}$ on the target. The target material was positioned $1 \mathrm{~mm}$ below the determined focus position in air, whereas the laser beam was focused by a $40 \mathrm{~mm}$ achromatic lens. The ablation process was performed in a 48-well plate, with a well size of $1.1 \mathrm{~cm}$ in diameter and $1.6 \mathrm{~cm}$ in height. The target was placed on the bottom of a well and covered with $500 \mu \mathrm{L}$ aqueous solution.
The well plate was placed on an axis-system (micro FS 150-2, 3D-Micromac AG) which moved at constant speed of $1 \mathrm{~mm} \mathrm{~s}^{-1}$ in a spiral (inner radius of $0.4 \mathrm{~mm}$, outer radius of $1 \mathrm{~mm}$ and line distance of $0.05 \mathrm{~mm}$ ) during the entire 159 seconds of irradiation period.

\subsection{In situ fabrication of AuNP-Penetratin mono- conjugates}

In order to fabricate bio-functionalized AuNP by in situ conjugation during laser ablation, we purchased the CPP penetratin (5'CRQIKIWFQNRRMKWKK3') from Panatecs $\mathrm{GmbH}$, with cysteine as electron donor moiety for directed AuNP conjugation. Adding $1 \mu \mathrm{M}$ penetratin solution prior to the ablation process (corresponding to $3 \times 10^{14}$ penetratin molecules), in situ conjugation is achieved during laser ablation as the thiol function of the cysteine coordinates the electron accepting AuNP with high affinity.

\subsection{In situ fabrication of AuNP-Penetratin-Aptamer bi-conjugates}

Synthesis of bi-functional bioconjugates was achieved by ablation of AuNP in a solution containing $1 \mu \mathrm{M}$ of TRITC-tagged CPP penetratin (5'CRQIKIWFQNRRMKWKK[TRITC]3') from Panatecs $\mathrm{GmbH}$ (corresponding to $3 \times 10^{14}$ penetratin molecules) and MiniStrep aptamer (5'-TCTGTGAGACGACGC ACCGGTCGCAGGTTTTGTCTCACAG-T10-( $\left(\mathrm{CH}_{2}\right)_{3}$-SS$\left.\left(\mathrm{CH}_{2}\right)_{3} \mathrm{OH}-3^{\prime}\right)$ from Operon Biotechnologies $\mathrm{GmbH}$ with specific binding to streptavidin in concentrations of $1.5 \mu \mathrm{M}$ or $4.5 \mu \mathrm{M}$, respectively (corresponding to $4.5 \times 10^{14}$ and $1.4 \times 10^{15}$ aptamer molecules, respectively). It is known, that aptamers may exhibit unspecific binding to AuNP surfaces resulting in a loss of aptamer activity. Therefore, an oligothymidine spacer between the miniStrep sequence and AuNP was chosen, since thymidine has the lowest affinity towards a gold surface in comparison to other possible nucleotides [34].

The target material was positioned $1.5 \mathrm{~mm}$ below the determined focus position in air and the time of irradiation was set to 106 seconds due to optimized ablation parameters for bi-conjugation. The ablation pattern was the equal spiral form as described in chapter 2.1.

\subsection{Fast ex situ surface functionalization}

Fast ex situ conjugation of AuNP was achieved by $6 \mathrm{~mJ}$, $3 \mathrm{kHz}$ nanosecond laser ablation (Rofin Sinar Laser $\mathrm{GmbH}$, RS-Marker 100D, 1064 nm, 40 ns FWHM) of gold in $2 \mathrm{~mL} / \mathrm{min} \mathrm{ddH}_{2} \mathrm{O}$ flow. Thiolated, Cy5-tagged CPP HIVTAT ([Cy5]GRKKRRQRRR-Ahx-C) from Panatecs $\mathrm{GmbH}$ (concentration: 1 - $40 \mu \mathrm{M}$ ) was introduced in a small microcapillary with equal flow rate, whereas the intersection was separated with a distance of $2 \mathrm{~mm}$ from the ablation zone. The schematic experimental description of this method is shown in figure 1. 


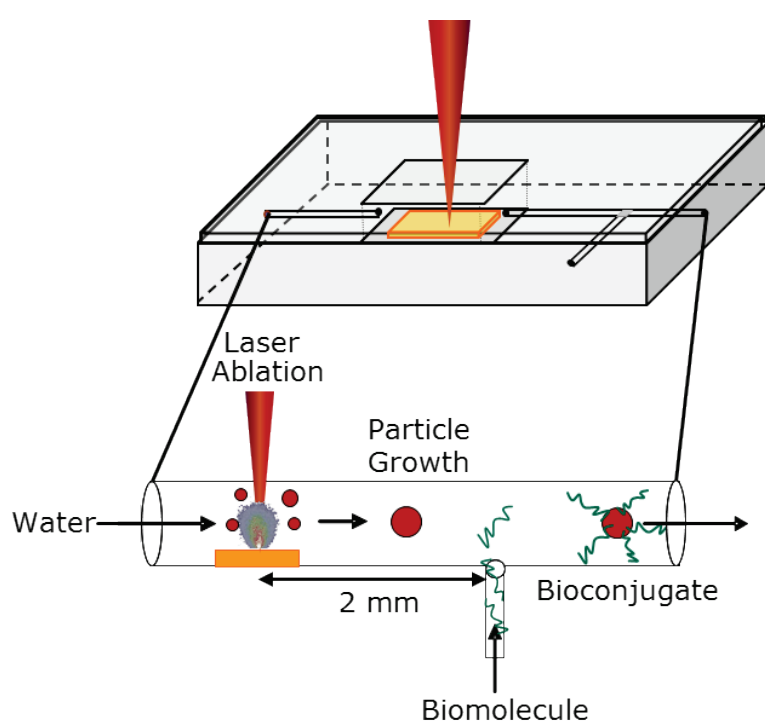

Figure 1: Scheme of the fast ex situ process allowing timedelayed conjugation of biomolecules to laser-generated gold nanoparticles in controlled liquid flow.

\subsection{AuNP and AuNP-bioconjugate characterization}

Optical extinction spectra of the colloidal solutions were measured using UV-vis spectroscopy with a Shimadzu UV-1650PC spectrograph in the spectral region of $200-800 \mathrm{~nm}$. Referring to a method from Muto et al. [35], the concentration of dispersed AuNP in solution in $\mu \mathrm{g} / \mathrm{mL}$ can be accurately determined using the absorbance intensity at $380 \mathrm{~nm}$. Therefore, the ablated mass of $\mathrm{Au}$ target was determined in triplicate for different ablation times using a Sartorius microgram balance ( $1 \mu \mathrm{g}$ accuracy) and correlated to the absorbance intensity at $380 \mathrm{~nm}$ in order to establish a standard calibration.

Transmission electron microscopy (TEM) analysis was performed on a $60 \mathrm{kV}$ EM $10 \mathrm{C}$ transmission electron microscope (Carl Zeiss AG). A drop of the sample solution was placed on a carbon-coated formvar-covered copper grid and dried at room temperature. The diameters of 500 particles were measured with the Image $\mathrm{J}$ software and classified to obtain an average Feret particle size distribution. Number of AuNP in solution was estimated using previously determined AuNP concentration, ablated mass and the mean Feret size of particles.

Conjugation efficiency and surface coverage of biomolecules on the AuNP were calculated after purification of conjugate solutions. To separate bioconjugated AuNP from unconjugated biomolecules, the samples were ultracentrifuged for $30 \mathrm{~min}$ at $40.000 \mathrm{rpm}$ on an Optima Max analytical ultracentrifuge (Beckmann Coulter $\mathrm{GmbH})$. The extinction of the biomoleculecontaining supernatant at $280 \mathrm{~nm}$ in case of unlabeled penetratin, or at $648 \mathrm{~nm}$ in case of Cy5-tagged HIV-TAT was measured and the unbound biomolecule concentration was interpolated from the extinction maximum of the standard calibration. For TRITC-tagged penetratin, the fluorescence (ext: $541 \mathrm{~nm}$, em: $572 \mathrm{~nm}$ ) of the supernatant was determined with a Fluroskan Ascent fluorometer (Thermo Fisher Scientific $\mathrm{GmbH}$ ) and the concentration of unbound biomolecule interpolated from a standard calibration. With regard to absorption of both miniStrep and penetratin in the UV-range, unbound concentration of miniStrep was determined by extinction at $260 \mathrm{~nm}$ and subtracted by the background absorption of penetratin at $260 \mathrm{~nm}$. Ratio of total and unconjugated biomolecule concentration in percent defined the conjugation efficiency of the biomolecules, while surface coverage was calculated by using distinct parameters of the AuNP like their average Feret size, density, mass and calculated surface area.

In order to study bioconjugate stability, $\xi$-potential measurements were systematically performed in an electrophoretic cell in triplicate with a Zetasizer ZS (Malvern Instruments).

\section{Results and discussion}

\subsection{AuNP-Penetratin mono-conjugates by in situ functionalization}

Using $1 \mu \mathrm{M}$ of penetratin solution for in situ conjugation, we fabricated AuNP-penetratin mono-conjugates by ultrashort-pulsed laser ablation. The obtained colloid is redcolored, stable with a $\xi$-potential value of $-25 \pm 1 \mathrm{mV}$ and the AuNP have an average Feret diameter of $7 \mathrm{~nm}$ as determined by TEM analysis (figure 2). Compared to ligand-free AuNP having a $\xi$-potential value of $-30 \pm 2 \mathrm{mV}$ and an average size of $9 \mathrm{~nm}$ with a broad size distribution (data not shown), the decrease of the mean nanoparticle size in combination with a distinct narrowing of the size distribution indicates surface functionalization, whereas size-reduction is achieved by nanoparticle growth quenching during nucleation and condensation of the ablation process. The decrease of $\xi$-potential for penetratinconjugates is caused by the conjugation of the positively net charged peptide to AuNP.

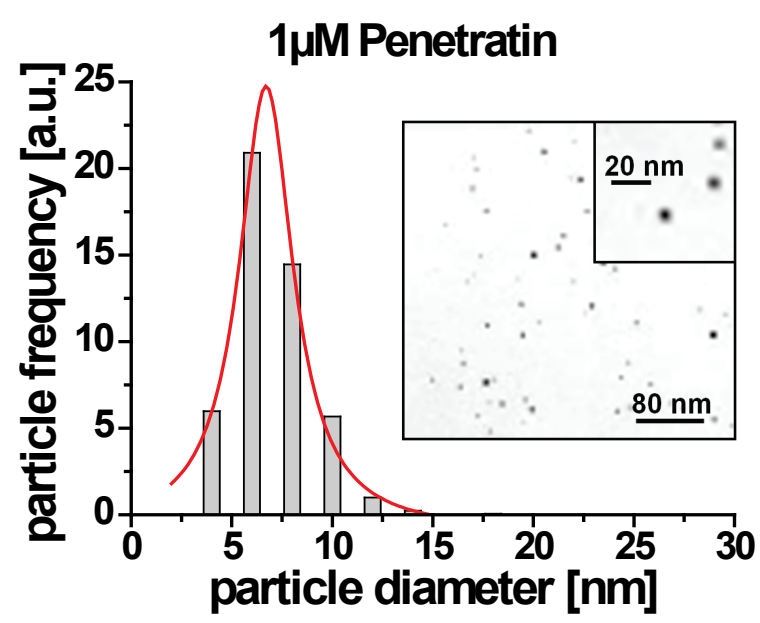

Figure 2: Size distribution of mono-functionalized AuNP with Lorentz fitting function (red line) and TEM micrograph (insert) of laser-generated AuNP functionalized with $1 \mu \mathrm{M}$ penetratin.

The conjugation efficiency of penetratin to AuNP is determined to be $84 \%$ (figure 4), indicating that a high amount of the total biomolecule number $\left(2.5 \times 10^{14}\right.$ molecules) has bound to the AuNP surface. Estimation of surface coverage assumes $38 \mathrm{pmol} / \mathrm{cm}^{2}$, equivalent to 
32 penetratin molecules per AuNP (figure 5). These data are comparable to the result of Petersen et al., who determined a surface coverage of 27 penetratin molecules per laser-generated AuNP using identical laser parameters and a slightly different nanoparticle-to-biomolecule ratio [36].

Since mono-functionalization of AuNP could be achieved easily and reproducible by in situ conjugation during laser ablation, we next aspired to obtain a bifunctionalization using the same synthesis method.

\subsection{AuNP-Penetratin-Aptamer bi-conjugates by in situ functionalization}

Besides laser-induced mono-conjugation, we achieved the conjugation of two different biomolecules to lasergenerated AuNP using the in situ functionalization method. $1 \mu \mathrm{M}$ penetratin and $1.5 \mu \mathrm{M}$ miniStrep solution resulted in a red-colored, stable colloid, referred to 'conjugate I' with a $\xi$-potential value of $-32 \pm 2 \mathrm{mV}$. Compared to penetratin mono-conjugated AuNP, the $\xi$-potential is shifted to the negative range due to the conjugation of the negatively net charged miniStrep aptamer. Accordingly, the conjugation with $1 \mu \mathrm{M}$ penetratin and $4.5 \mu \mathrm{M}$ aptamer generated a biconjugate colloid referred to 'conjugate II' with an even elevated negative $\xi$-potential value of $-42 \pm 1 \mathrm{mV}$ due to the increased number of negatively net charged miniStrep aptamer molecules on the AuNP.

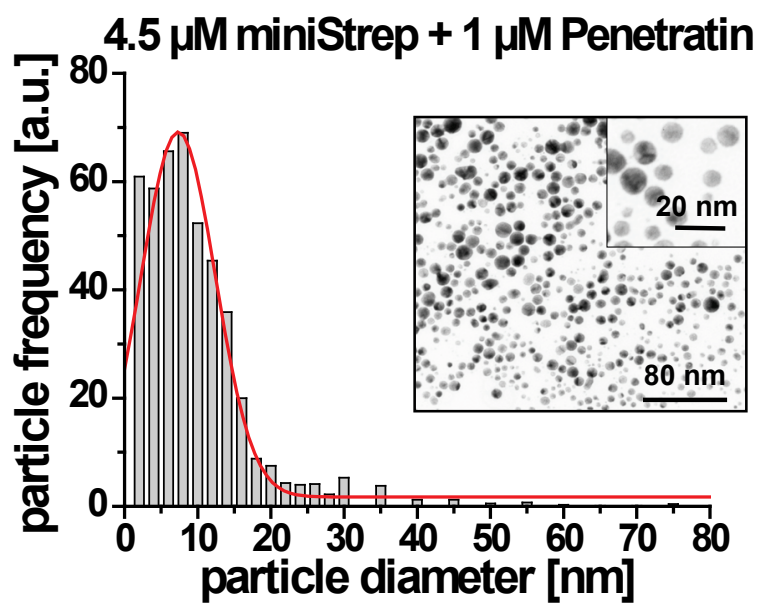

Figure 3: Size distribution of bi-functionalized AuNP with Gaussian fitting function (red line) and TEM micrograph (insert) of laser-generated AuNP with $1 \mu \mathrm{M}$ penetratin and $4.5 \mu \mathrm{M}$ miniStrep.

As determined by TEM analysis, the bi-conjugated AuNP are spherical and well-separated with an average Feret diameter of $10 \mathrm{~nm}$ (figure 3). No size quenching effect is visible compared to size distribution of ligand-free AuNP. This fact is due to electrosterical binding hindrance between the positive (penetratin) and negative (miniStrep) net-charged biomolecules on the AuNP surface, so that the nanoparticles are able to grow unquenched during the nucleation and condensation steps of the ablation process.

Conjugation efficiencies of biomolecules for 'conjugate I' were determined to be $47 \%$ for miniStrep and $99 \%$ for penetratin, respectively (figure 4). These values correspond to $2.97 \times 10^{14}$ penetratin molecules and $2.11 \times 10^{14}$ miniStrep molecules in total. Compared to the monofunctionalized AuNP-penetratin conjugate, the conjugation efficiency of penetratin is higher referring to slightly optimized laser parameters (target position: $1.5 \mathrm{~mm}$ below the determined focus position in air, irradiation time: 106 seconds) as well as to the associated nanoparticle-tobiomolecule ratio and biomolecule integrity. We found the conjugation efficiency of miniStrep being lower than that for penetratin, possibly due to larger molecule size caused by steric folding, which has an important impact on binding affinity. Using the online RCSB protein data base and the Jmol software we determined the steric dimensions of the penetratin molecule (ID: $1 \mathrm{KZ} 0$ ) to be around $1.6 \mathrm{~nm} \times 1.5 \mathrm{~nm}$ in width, while online Mfold software predicted miniStrep aptamer to fold into a stem-loop structure with a maximum width of $5.5 \mathrm{~nm} \mathrm{x} 4 \mathrm{~nm}$ (assuming base length of $0.33 \mathrm{~nm}$ ). In consequence, the voluminous miniStrep is sterically hindered to bind as effective to the AuNP surface as the small penetratin peptide. Moreover, nanoparticles may reject the conjugation of the negatively charged aptamer biomolecule due to their own negative $\xi$-potential.

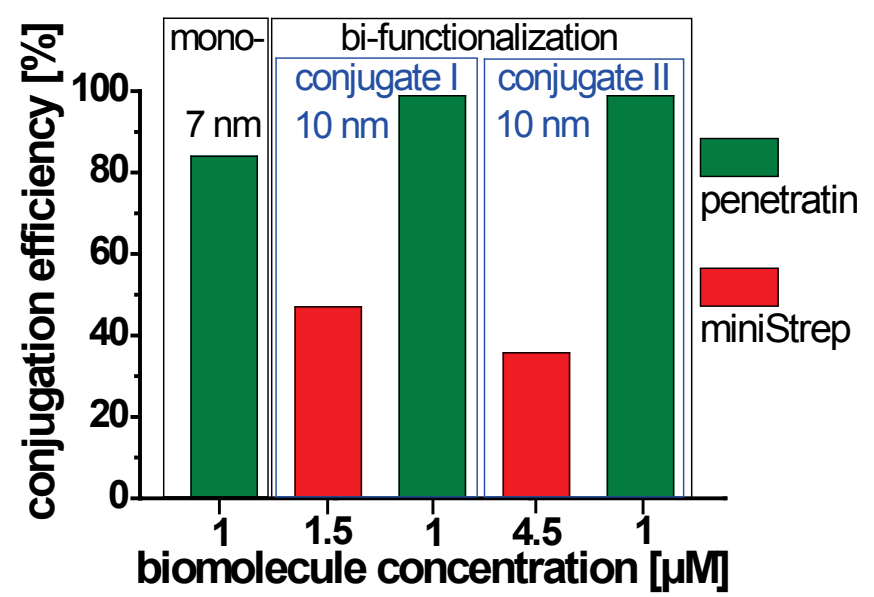

Figure 4: Conjugation efficiencies of in situ monofunctionalized AuNP (7 $\mathrm{nm}$ average size) with $1 \mu \mathrm{M}$ penetratin and in situ bi-functionalized AuNP (10 nm average size) with $1 \mu \mathrm{M}$ penetratin and $1.5 \mu \mathrm{M}$ and $4.5 \mu \mathrm{M}$ miniStrep aptamer, referred to 'conjugate I' and 'conjugate II', respectively.

Calculating the surface coverage values for 'conjugate I' we determined $32 \mathrm{pmol} / \mathrm{cm}^{2}$ for miniStrep and 33 $\mathrm{pmol} / \mathrm{cm}^{2}$ for penetratin respectively (figure 5), corresponding to a total number of 122 biomolecules on the nanoparticle surface. In detail, 66 penetratin molecules and 56 aptamer molecules were attached to a single nanoparticle surface, illustrating a nearly homogenous distribution of both biomolecules. However, the surface coverage value of penetratin is lower for bi-functionalized particles than for the mono-functionalized ones, due to lower AuNP concentration and the increased AuNP Feret size and particle surface area. 


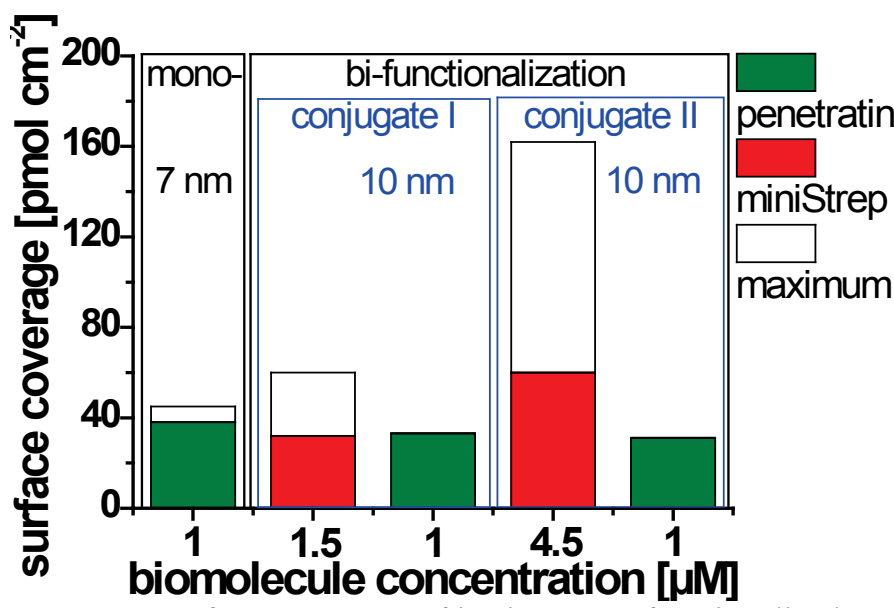

Figure 5: Surface coverages of in situ mono-functionalized AuNP ( $7 \mathrm{~nm}$ average size) with $1 \mu \mathrm{M}$ penetratin and in situ bi-functionalized AuNP (10 nm average size) with $1 \mu \mathrm{M}$ penetratin and $1.5 \mu \mathrm{M}$ and $4.5 \mu \mathrm{M}$ miniStrep aptamer referred to 'conjugate I' and 'conjugate II', respectively. Calculated maximum values are defined as maximal feasible surface coverages in case that all biomolecules have bound to the surface.

Evaluating the bi-functionalized 'conjugate II', we detected the same conjugation efficiency for penetratin of $99 \%$. However, compared to 'conjugate I' the conjugation efficiency for miniStrep is lowered to $37 \%$ (corresponding to $5 \times 10^{14}$ miniStrep molecules) in cause of the enhanced biomolecule concentration. Since there is a three times higher number of aptamer biomolecules in solution than for 'conjugate I' and since the surface area of AuNP is limited, a lower amount of total biomolecule number is able to bind to the particles. However, the number of conjugated miniStrep is twice higher for 'conjugate II' than for 'conjugate I' $\left(5 \times 10^{14}\right.$ compared to $\left.2.11 \times 10^{14}\right)$, resulting in a higher surface coverage of $61 \mathrm{pmol} / \mathrm{cm}^{2}$, while the surface coverage for penetratin is constantly $30 \mathrm{pmol} / \mathrm{cm}^{2}$. These values correspond to a conjugation of 172 biomolecules in total on the nanoparticle surface, which are classified into 66 penetratin molecules and 106 aptamer molecules. We suppose that the enhanced conjugation of miniStrep molecules is due to a core-shellshell multi-layer structure (Au-penetratin-miniStrep) and attachment of the negatively net charged aptamer molecule to the positively net charged penetratin.

These results illustrate the variability in ligand design and the tuning of AuNP surface coverage by variation of the biomolecule concentration. With this in situ conjugation method we are able to generate bi-functionalized gold nanoparticles with different biomolecules and defined surface coverages for customized applications. However, exploring the functional activity of the nano-bioconjugates is still subject of further investigations.

In prospect of process up-scaling, a time-delayed bioconjugation was investigated in a liquid flow chamber set-up using a biomolecule concentration series.

\subsection{AuNP-HIV-TAT mono-conjugates by fast ex situ} functionalization

According to the literature, conjugation efficiency and degree of surface coverage depend exponentially on the concentration of conjugant, as surface functionalization is limited by the effective nanoparticle surface [35].

Using the novel fast ex situ conjugation technique, schematically presented in figure 1, $6 \mathrm{~mJ}$ nanosecond laser pulses yielded about $1 \mathrm{mg} / \mathrm{min}$ ligand-free AuNP with a mean particle Feret size of $30 \mathrm{~nm}$ (Figure 7a).

Figure 6 depicts the determined conjugation efficiencies for nanosecond laser ablation of gold in water, followed once by ex situ bioconjugation in batch and once by the fast ex situ bioconjugation in a micro-fluidic flow chamber. We found the fast ex situ surface functionalization in liquid flow resulting in a 10 to $20 \%$ higher degree of conjugation, as ligand absorption is induced while the nanoparticles are still in the early post-growth phase, similar as during in situ conjugation method. Even at very high peptide concentration of $50 \mu \mathrm{M}$, a conjugation efficiency of $12 \%$ was achieved in liquid flow compared to only $2 \%$ achieved by ex situ surface functionalization in batch process.

Since conventional ex situ bioconjugation of nanoparticles is known to cause agglomeration effects on the nanoparticles and regarding applicability of the fast ex situ generated conjugates, we compared TEM micrographs for pure AuNP samples to $2.5 \mu \mathrm{M}$ and $15 \mu \mathrm{M}$ HIV-TAT concentrations. As demonstrated in Figure 7, the agglomeration rate and Feret size of nanoparticles changed significantly with biomolecule concentration. For $2.5 \mu \mathrm{M}$ HIV-TAT, a mean particle Feret size of $64 \mathrm{~nm}$ was determined while $15 \mu \mathrm{M}$ HIV-TAT concentrations resulted in a mean Feret size of $23 \mathrm{~nm}$. These results apply that a minimum number of ligands is required for effective stabilization of the fabricated AuNP and to induce the size quenching effect. Below the critical concentration, nanoparticle growth is promoted by ultrasmall gold entities such as atoms, clusters or ions in solution and interparticular aggregation is favored [28].

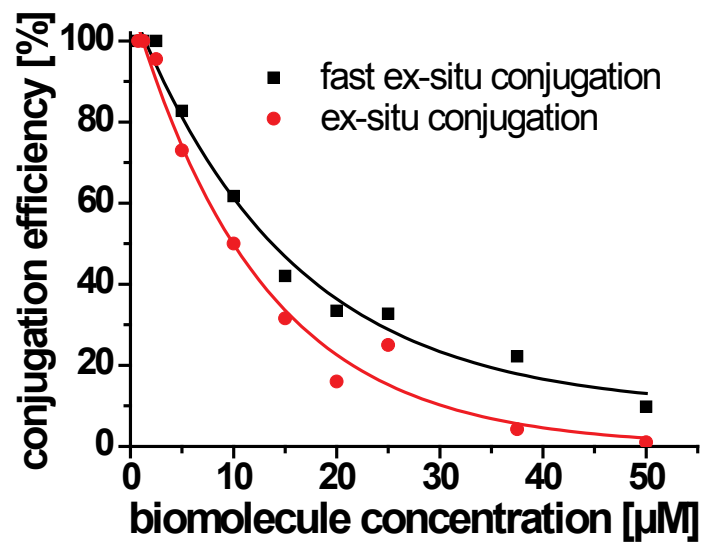

Figure 6: Conjugation efficiency of laser-generated gold nanoparticles by Cy5-tagged HIV-TAT cell penetrating peptides of various concentrations achieved by ex situ (batch) and fast ex situ (flow) bioconjugation. 
Thus, we note that efficient bioconjugation of AuNP may be achieved using high power laser irradiation without inducing photo-thermal degradation of biomolecules. However, critical concentration of ligands is required for conjugate applicability.
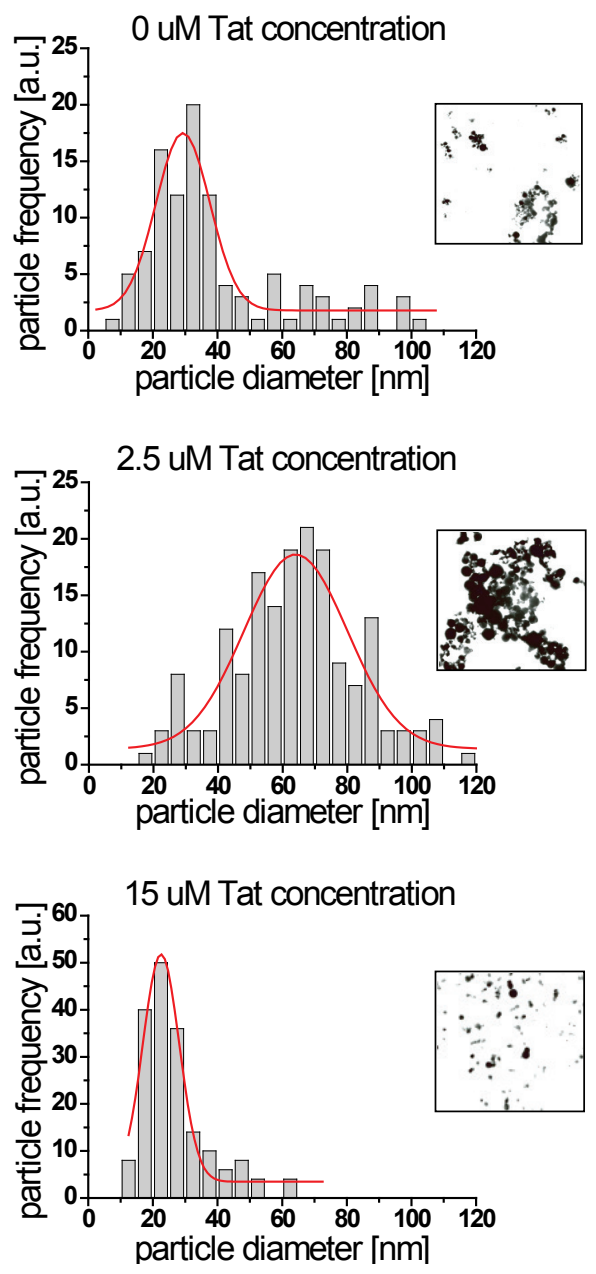

Figure 7: Unconjugated and HIV-TAT-conjugated AuNP by fast ex situ functionalization: nanoparticle size distributions with Lorentz fitting functions (red lines) and TEM micrographs (inserts) of laser-generated AuNP with $0 \mu \mathrm{M}, 2.5 \mu \mathrm{M}$ and $15 \mu \mathrm{M}$ HIV-TAT concentrations, respectively.

\section{Conclusion}

Gold nanoparticle surface functionalization is introduced both by using femtosecond- and nanosecond-pulsed laser ablation in liquids. Femtosecond laser-induced in situ functionalization and nanosecond laser-based fast ex situ conjugation within liquid flow for up-scaling purpose are presented for the first time to generate mono- and bifunctional AuNP-bioconjugates with ligand variability in biomolecules containing an electron-donor moiety. Streptavidin-targeted miniStrep aptamer and the cellpenetrating peptides penetratin and HIV-TAT were effectively coupled to AuNP, whereas conjugation efficiency and degree of surface coverage was deeply investigated. The laser-generated, spherical AuNP mono- or bi-conjugate colloids feature high stability and tunable size distribution due to biomolecule selection and concentration. Conjugation efficiencies and surface coverage values of the biomolecules can be controlled using different molar concentrations and biomolecule sizes. Exploiting the highpower laser irradiation in combination with a liquid flow fast ex situ conjugation process, the efficiency of nanobioconjugate generation may be increased even without inducing photo-thermal degradation of the biomolecules.

According to this fundamental work, we plan to generate customized, multi-functional AuNP bioconjugates with high efficiency and both, individual ligand combinations as well as tunable surface coverage ratios, addressing multitude biomedical applications.

\section{Acknowledgements}

This work was funded by the German Research Foundation within the excellence cluster REBIRTH. The authors thank Dr. Falk Hartmann, PD Ute Curth and Prof. Dietmar Manstein (Medizinische Hochschule Hannover) for the use of the ultracentrifugation facilities and Kerstin Rohn (Tierärztliche Hochschule Hannover) for transmission electron microscopy analysis.

\section{References}

[1] N. Lewinski, V. Colvin, and R. Drezek: small, 4(1) (2008) 26-49.

[2] I. H. El-Sayed, X. H., and M. A. El-Sayed: Nano Lett., 5(5) (2005) 829-834.

[3] Y.-F. Huang, Y.-W. Lin, Z.-H. Lin and H.-T. Chang: J Nanopart Res, 11 (2009) 775-783.

[4] G. Han, P. Ghosh, M. De and V. M. Rotello: Nanobiotech, 2 (2007) 40-45.

[5] K. Xiao, J. Luo, W. L, Fowler, Y. Li, J.S. Lee, L. Xing, R. H. Cheng, L. Wang, and K. S. Lam: Biomaterials, 30 (2009) 6006-6016.

[6] P. K. Jain, I. H. El-Sayed and M. A. El-Sayed: Nano Today, 2 (2007) 18-29.

[7] J. Wang, A. Munir and H. S. Zhou: Talanta, 79 (2009) $72-76$.

[8] A. G. Tkachenko, H. Xie, Y. Liu, D. Coleman, J. Ryan, W. R. Glomm, M. K. Shipton, S. Franzen and S. Feldheim: Bioconjugate Chem., 15 (2004) 482-490.

[9] C. C. Berry and J. M. La Fuente: Proc. SPIE, 7189 (2009) 71890U-71890U-11.

[10] S. Petersen, A. Barchanski, U. Taylor, S. Klein, D. Rath and S. Barcikowski, J. Phys. Chem. C, 115 (2011) 5152-5159.

[11] S. Petersen and S. Barcikowski: Adv. Funct. Mater., 19 (2009) 1-6.

[12] J. G. Walter, S. Petersen, F. Stahl, T. Scheper, S. Barcikowski: J. Nanobiotech., 8:21, doi:10.1186/14773155-8-21, 2010.

[13]Z. Medarova, W. Pham, C. Farrar, V. Petkova, and A. Moore: Nat. Med., 13(3) (2007) 372-377.

[14] O. C. Farokhzad, J. Cheng, B. A. Teply, I. Sherifi, S. Jon, P.W. Kantoff, J.P. Richie, and R. Langer: PNAS, 103 (2006) 6315-6320.

[15] J. D. Gibson, B. P. Khanal and E. R. Zubarev: J. Am. Chem. Soc., 129 (2007) 11653-11661. 
[16] M. Brust, M. Walker, D. Bethell, D. J. Schiffrin, and R. Whyman: J. Am. Chem. Soc. Chem. Comm., 801(1994) 801-802.

[17] J. Turkevich, P. C. Stevenson, and J. Hillier: Disc. Faraday Soc., 11 (1951) 55-75.

[18] A. Henglein: J. Phys. Chem., 97 (1993) 5457-5471.

[19] V. Amendola, and M. Meneghetti: Phys Chem Chem Phys, 11(20) (2009) 3805-3821.

[20] S. Barcikowski, A. Hahn, A. V. Kabashin, and B. N. Chichkov: Appl. Phys. A, 87(1) (2007) 47-55.

[21] K. M. Stewart, K. L. Horton, and K. O. Shana: Org. Biomol. Chem., 6(6) (2008) 2242-2255.

[22] C. C. Berry, J. M. La Fuente, M. Mullin, S. W. L. Chu, and A. S. G. Curtis: IEEE Trans. Nanobiosci., 6(4) (2007) 262-269.

[23] G. Dom, C. Shaw-Jackson, C. Matis, O. Bouffioux, J. J. Picard, A. Prochiantz, M.-P. Mingeot-Leclercq, R. Brasseur, and R. Rezsohazy: Nucleic Acids Res., 31 (2003) 556-561.

[24]F. Duchardt, M. Fotin-Mleczek, H. Schwarz, R. Fischer, and R. Brock: Traffic, 8(7) (2007) 848-866.

[25]D. Derossi, S. Calvet, A. Trembleau, A. Brunissen, and G. Chassaing: J. Biol. Chem., 271 (1996) 1818818193.

[26]M. Green, and P. M. Loewenstein: Cell, 55 (1998) 1179-1188.

[27] J. M. de la Fuente, and C. C. Berry: Bioconj. Chem., 16 (2005) 1176-1180.

[28] C. L. Sajti, A. Barchanski, P. Wagener, S. Klein, S. Barcikowski: J. Phys. Chem. C., 115(12) (2011) 50945101.

[29] V. Pavlov, Y. Xiao, B. Shlyahovski, and I. Willner: J. Am. Chem. Soc., 126(38) (2004) 11768-11769.

[30] J. Liu, and Y. Lu: Nat. Prot., 1(1) (2006) 246-252.

[31] H. Xu, X. Mao, Q. Zeng, S. Wang, A. N. Kawde, and G. Liu: Anal. Chem., 81(2) (2009), 669-675.

[32] J. G. Walter, O. Kokpinar, K, Friehs, F. Stahl, and T. Scheper: Anal. Chem., 80(19) (2008) 7372-7378.

[33] C. L. Sajti, S. Petersen, A. Menéndez-Manjón, S. Barcikowski: Appl. Phys. A: Mat. Sci. \& Proc., 101(2) (2010) 259-264.

[34] H. Kimura-Suda, D.Y. Petrovykh, M.J. Tarlov, and L.J. Whitman: J. Am. Chem. Soc., 125(30) (2003) 90149015.

[35]H. Muto, K. Yamada, K. Miyajima, F. Mafume: J. Phys. Chem. C, 111 (2007) 17221-17226.

[36] S. Petersen, and S. Barcikowski: J. Phys. Chem. C, 113(46) (2009) 19830-19835.

(Received: June 07, 2010, Accepted: June 13, 2011) 